\title{
PKLot - A Robust Dataset for Parking Lot Classification
}

\author{
Paulo Almeida and Luiz S. Oliveira \\ Universidade Federal do Paraná (UFPR), Curitiba, PR, Brazil \\ paulolra@gmail.com, lesoliveira@inf.ufpr.br \\ Alceu S. Britto Jr, Eunelson J. Silva Jr, and Alessandro Koerich \\ Pontifícia Universidade Católica do Paraná (PUCPR), Curitiba, PR, Brazil \\ Universidade Estadual de Ponta Grossa(UEPG), Ponta Grossa, PR, Brazil \\ \{alceu, eunelson, alekoe\}@ppgia.pucpr.br
}

\section{Introduction}

A major challenge to pursuing research involving parking space classification is the lack of a consistent and reliable dataset. To overcome this problem, the main contribution of this work is to present the PKLot, a robust image dataset of parking lots which is an extended version of the one introduced in Almeida2013. It was extended from 105,837 to 695,899 images. In this new version, the parking spaces were captured from different parking lots under varied weather conditions. Each parking space image was manually checked and classified according to its situation (vacant or occupied) and to the weather condition observed during the image acquisition (sunny, overcast or rainy). The PKLot is available for research purposes under request ${ }^{1}$.

\section{The PKLot Dataset}

The PKLot dataset contains 12,417 images of parking lots and 695,899 images of parking spaces segmented from them, which were manually checked and labeled. All images were acquired at the parking lots of the Federal University of Parana (UFPR) and the Pontifical Catholic University of Parana (PUCPR), both located in Curitiba, Brazil. The protocol used to construct the PKLot dataset is composed of three steps, as follows:

- Image Acquisition: this process was defined to be executed with a 5-minute time-lapse interval for a period of more than 30 days by means of a low cost full HD camera (Microsoft LifeCam, HD-5000) positioned at the top of a building to minimize the possible occlusion between adjacent vehicles. The main goal was to obtain images under different weather conditions (overcast, sunny, and rainy periods) by registering at each 5 minutes the environment changes. Such a setup, allows to capture sequences of images showing high variability in terms of illumination occasioned by weather changes. For instance, in a short period it is possible to observe light rain, heavy rain, and after rain conditions. Unfortunately, we do not have night shots since the illumination available in the parking lots was not sufficient to acquire good quality images. The resulting images were stored in JPEG color format with lossless compression (quality 100\%) in a resolution of $1280 \times 720$ pixels. They were organized into three subsets named UFPR04, UFPR05 and PUCPR. The first two contain images of different views of the same parking lot captured from the 4th and 5th floors of the UFPR building. The last dataset contains images captured from the 10th floor of the administration building of the PUCPR. Figure 1 shows some image samples of the three parking lots captured under the aforementioned weather conditions. It is possible to observe some challenges posed by this dataset, such as: sunny images (Figure 1a) presenting overexposed cars and shadows caused by the trees; or images acquired under heavy rain (Figure1c) that look like night images due the lack of natural light.

${ }^{1}$ http://web.inf.ufpr.br/vri/parking-lot-database 
- Labeling: for each parking lot image was created an Extensible Markup Language (XML) file containing the position and situation (vacant or occupied) of each parking space. An interactive tool was developed to label the images. Such a tool allows the visualization of each image and the definition of the limits of each parking space (represented by points of a polygon), as well as the situation (vacant or occupied). Different subfolders were used to manually categorize the images according to the observed weather condition (overcast, sunny, or rainy period).

- Segmentation: the individual parking spaces were extracted from each parking lot image using the information available in the corresponding XML file. In addition, the slope of the rectangle containing the parking space image was modified according to the scheme presented in Figure 2. Those with less than $45^{\circ}$ were rotated to $0^{\circ}$, while those with more than $45^{\circ}$ were rotated to $90^{\circ}$. Figure 3 a shows an image where the 28 available spaces are marked in green. It is important to mention that only valid parking spaces were labeled and segmented. Valid parking spaces are those signed (delimited) with parallel yellow or white lines on the floor. As one can notice, there are some cars parked in an unauthorized manner, i.e., in the middle of the street. Two samples of the segmented parking spaces are depicted in Figures $3 \mathrm{~b}$ (occupied) and 3c (empty).

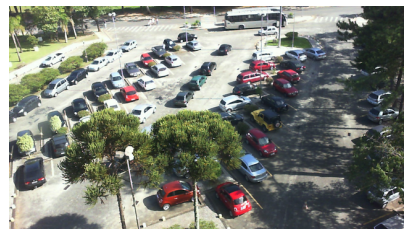

(a)

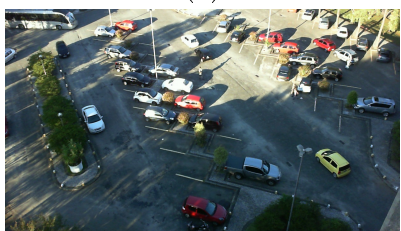

(d)

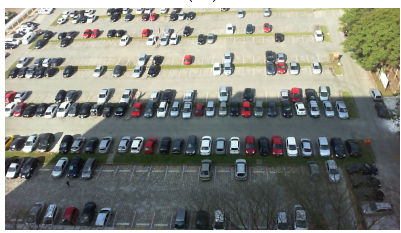

(g)

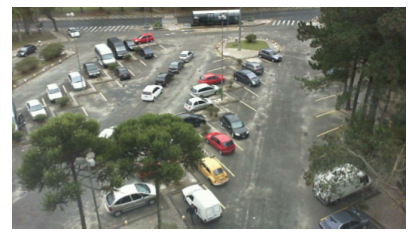

(b)

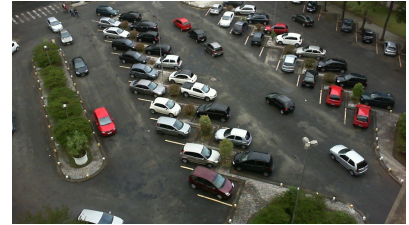

(e)

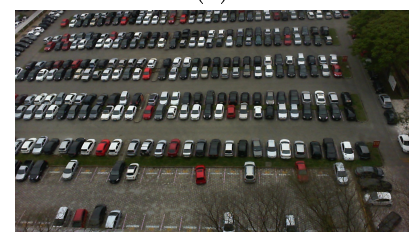

(h)

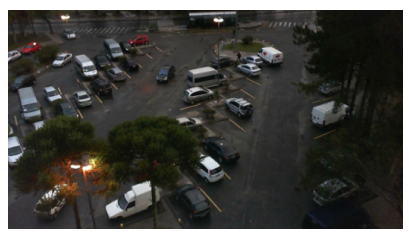

(c)

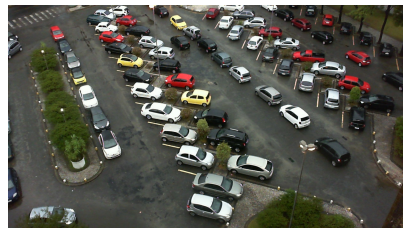

(f)

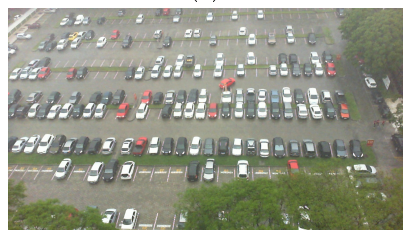

(i)

Figure 1: Images captured under different weather conditions: (a) sunny (b) overcast, and (c) rainy from UFPR04; (d) sunny (e) overcast, and (f) rainy from UFPR05; and (g) sunny (h) overcast, and (i) rainy from PUCPR

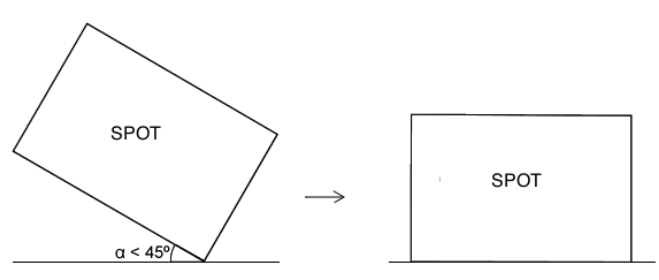

(a)

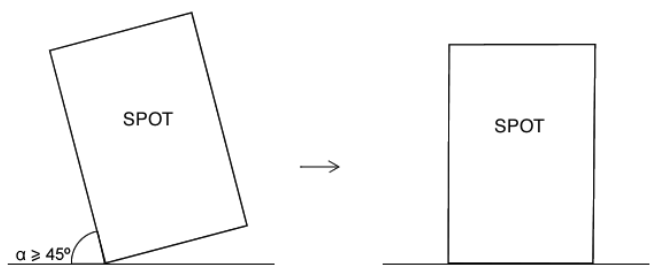

(b)

Figure 2: Skew adjustment

Table 1 summarizes the general characteristics of each subset. As one may see, for instance, the images captured from the subset UFPR04 allow us to monitor 28 individual spaces. This 


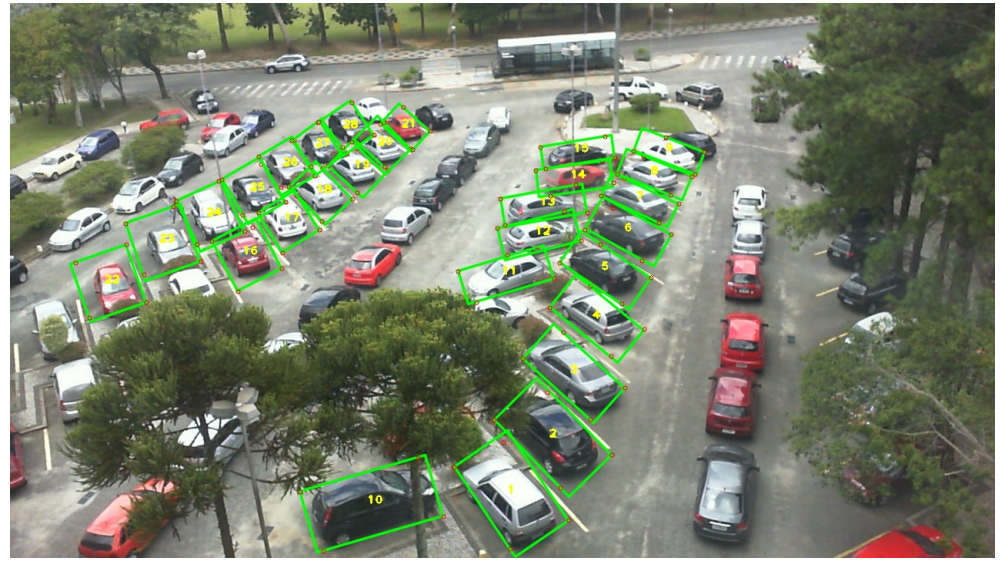

(a)

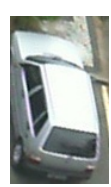

(b)

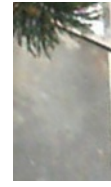

(c)

Figure 3: Segmented image: (a) 28 delimited spaces, (b) occupied sub-image, and (c) empty sub-image.

subset contains 3,791 images captured under different weather conditions. After a semi-automatic segmentation process and a manual checking and labeling, it sums up 105,845 images of individual lots, $43.48 \%$ occupied and $56.42 \%$ empty. Similar information is provided for the UFPR05 and PUCPR subsets.

Here is a short summary of what makes this dataset interesting to computer vision research community:

(i) images covering different climatic conditions (sunny, rainy and overcast periods) were taken under uncontrolled illumination;

(ii) images were taken from different parking lots presenting distinct features;

(iii) cameras were positioned at different heights;

(iv) images show a varied kind of problems, such as the presence of shadows, over-exposition to sunlight, low light in rainy days, difference in perspective, and so on;

(v) vehicle images are typical for a commercial surveillance system, i.e. the camera is placed highly above the vehicles, making the detection even more demanding;

(vi) a large number of potential uses.

Although the first and the most important potential use of this dataset is to test the robustness of algorithms with the objective of detecting vacant parking spaces in a real-world surveillance scenario, its application is not restricted to it. Our cameras were placed at different heights and the images were gathered at different climatic conditions. This issue is the strongest point of this dataset. It remains to be seen how will algorithms perform in such variable conditions and how does the vehicle distance from camera influence the results. There is also a potential to test various image preprocessing algorithms, as some of parking spaces are far from the camera mounting position and the perspective plays an important role in such cases.

In addition, by including different pose images of vehicles and parking spaces, we made it possible to use this dataset in modeling parking spaces and vehicles. Other potential uses of this dataset include but are not restricted to: evaluation of algorithms' robustness to different vehicle poses, evaluation of natural illumination normalization algorithms, evaluation of new features for vehicle (or space) description, and so on.

\section{Proposed Evaluation Protocol}

This section describes an evaluation protocol proposed on the basis of the PKLot dataset, which is applied in the experiments presented in Section 5. 
Table 1: Summary of the PKLot characteristics

\begin{tabular}{llrrrrr}
\hline \multirow{2}{*}{$\begin{array}{c}\text { Parking } \\
\text { lot }\end{array}$} & Weather & \# of & \# of & \multicolumn{2}{c}{ \# of parking spaces } & \\
\cline { 6 - 7 } condition & days & images & occupied & empty & total \\
\hline UFPR04 & Sunny & 20 & 2,098 & $32,166(54.98 \%)$ & $26,334(45.02 \%)$ & 58,400 \\
(28 parking spaces) & Overcast & 15 & 1,408 & $11,608(29.47 \%)$ & $27,779(70.53 \%)$ & 39,387 \\
& Rainy & 14 & 285 & $2,351(29.54 \%)$ & $5,607(70.46 \%)$ & 7,958 \\
& Subtotal & & 3,791 & $46,125(43.58 \%)$ & $59,720(56.42 \%)$ & 105,845 \\
\hline UFPR05 & Sunny & 25 & 2,500 & $57,584(57.65 \%)$ & $42,306(42.35 \%)$ & 99,890 \\
(45 parking spaces) & Overcast & 19 & 1,426 & $33,764(59,27 \%)$ & $23,202(40.73 \%)$ & 56.966 \\
& Rainy & 8 & 226 & $6,078(68.07 \%)$ & $2,851(31.93 \%)$ & 8,929 \\
& Subtotal & & 4,152 & $97,426(58.77 \%)$ & $68.359(41.23 \%)$ & 165,785 \\
\hline PUCPR & Sunny & 24 & 2,315 & $96,762(46.42 \%)$ & $111,672(53.58 \%)$ & 208,433 \\
(100 parking spaces) & Overcast & 11 & 1,328 & $42,363(31.90 \%)$ & $90,417(68.10 \%)$ & 132,780 \\
& Rainy & 8 & 831 & $55,104(66,35 \%)$ & $27,951(33.65 \%)$ & 83,056 \\
& Subtotal & & 4,474 & $194,229(45.78 \%)$ & $230.040(51.46 \%)$ & 424,269 \\
\hline TOTAL & & & 12,417 & $337,780(48.54 \%)$ & $358.119(51.46 \%)$ & 695.899
\end{tabular}

Table 2: Training and Testing Sets

\begin{tabular}{|l|l|r|r|r||r|r|r|}
\cline { 3 - 8 } \multicolumn{2}{c|}{} & \multicolumn{3}{c||}{ Training sets } & \multicolumn{3}{c|}{ Testing sets } \\
\cline { 3 - 8 } \multicolumn{2}{c|}{} & Occupied & Empty & Total & Occupied & Empty & \multicolumn{1}{c|}{ Total } \\
\hline \multirow{3}{*}{ UFPR04 } & Sunny & 16,524 & 14,327 & 30,851 & 15,642 & 12,007 & 27,649 \\
& Overcast & 6,989 & 15,076 & 22,065 & 4,619 & 12,703 & 17,322 \\
& Rainy & 1,041 & 2,553 & 3,594 & 1,310 & 3,054 & 4,364 \\
& Total & $\mathbf{2 4 , 5 5 4}$ & $\mathbf{3 1 , 9 5 6}$ & $\mathbf{5 6 , 5 1 0}$ & $\mathbf{2 1 , 5 7 1}$ & $\mathbf{2 7 , 7 6 4}$ & $\mathbf{4 9 , 3 3 5}$ \\
\hline \hline \multirow{3}{*}{ UFPR05 } & Sunny & 28,822 & 21,657 & 50,479 & 28,762 & 20,649 & 49,411 \\
& Overcast & 15,421 & 12,985 & 28,406 & 18,343 & 10,217 & 28,560 \\
& Rainy & 2,751 & 1,633 & 4,384 & 3,327 & 1,218 & 4,545 \\
& Total & $\mathbf{4 6 , 9 9 4}$ & $\mathbf{3 6 , 2 7 5}$ & $\mathbf{8 3 , 2 6 9}$ & $\mathbf{5 0 , 4 3 2}$ & $\mathbf{3 2 , 0 8 4}$ & $\mathbf{8 2 , 5 1 6}$ \\
\hline \hline \multirow{3}{*}{ PUCPR } & Sunny & 47,490 & 59,731 & 107,221 & 49,271 & 51,941 & 101,212 \\
& Overcast & 26,774 & 42,933 & 69,707 & 15,589 & 47,484 & 63,073 \\
& Rainy & 19,540 & 16,025 & 35,565 & 35,565 & 11,926 & 47,491 \\
& Total & $\mathbf{9 3 , 8 0 4}$ & $\mathbf{1 1 8 , 6 8 9}$ & $\mathbf{2 1 2 , 4 9 3}$ & $\mathbf{1 0 0 , 4 2 5}$ & $\mathbf{1 1 1 , 3 5 1}$ & $\mathbf{2 1 1 , 7 7 6}$ \\
\hline \hline
\end{tabular}

\subsection{Definition of Training/Testing Sets}

The strategy used to select images to compose the training and testing sets follows an important rule in the PKLot dataset. This rule determines that images of the same day can belong to just one of these sets. This avoids that pictures related to the same car parked in the same space for hours showing just light variations can appear in the training and the testing sets simultaneously. With this in mind, we suggest in this protocol to consider $50 \%$ of the images available in the subsets UFPR04, UFPR05 and PUCPR for training and 50\% for testing. Table 2 shows the amount of samples available for training and testing, which follows this proportion.

\subsection{Performance Estimation}

The performance of the created classifiers on the testing set can be estimated based on the Overall Error Rate given by Equation 1.

$$
\text { Overall Error Rate }=\frac{F P+F N}{T P+T N+F P+F N}
$$


where $F P, F N, T P$, and $T N$ stand for False Positive, False Negative, True Positive, and True Negative, respectively. These statistics can be defined in the $2 \times 2$ confusion matrix depicted in Figure 4 .

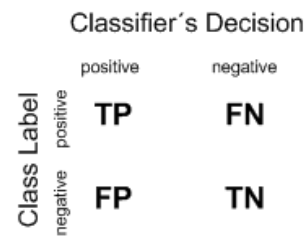

Figure 4: $2 \times 2$ confusion matrix.

Another interesting tool for performance estimation is the Receiver Operating Characteristics (ROC) curve. It is created by plotting the $T P$ rate (sensitivity) against the $F P$ rate (specificity) at various threshold settings. ROC analysis provides tools to select possibly optimal models. An interesting measure of the accuracy is the area under the ROC curve. A test is $100 \%$ accurate if both the sensitivity and specificity are 1.0. It means that there are no false positives and no false negatives.

\subsection{Research Directions}

Different research directions can be followed on the basis of the PKLot dataset, as follows:

- Single Parking Lot Training and Testing: This experiment is devoted to evaluate the suitability of feature sets to represent the presence or absence of a vehicle in a parking space as well as to compare the performance of classifiers trained on descriptors extracted from images that belong to a single parking lot. It means that each classifier is trained on images from one of the training subsets (UFPR04, UFPR05 or PUCPR) and assessed using the respective testing set. For instance, considering UFPR04 as training set, the accuracies and confusion matrices related to the each classifier are computed only for the UFPR04 testing set.

- Single Parking Lot Training and Multiple Parking Lot Testing: This experiment is devoted to measure the generalization power of features and classifiers. For such an aim, classifiers trained on images of a specific parking lot are used to classify images from other parking lots. It means that each classifier is trained on images from one of the training subsets (UFPR04, UFPR05 or PUCPR), however they are assessed using a different testing set. Thus, considering UFPR04 as training set, the accuracies and confusion matrices related to the each classifier are computed for the testing sets available in UFPR05 and PUCPR.

- Multiple Parking Lot Training: here, the classifiers are trained on images of multiple parking lots. Thus, the objective is to measure the ability of the trained classifiers in absorbing the wide variability related to the images captured using different angles of view and camera height mounting as well as presenting varied surface patterns. It means that each classifier is trained on images from two or more of the training subsets (UFPR04, UFPR05 and PUCPR), however they are assessed using a single testing set. Thus, considering the fusion of UFPR04 and UFPR05 as training set, the accuracies and confusion matrices related are computed for the testing set available in PUCPR. In order to produce a balanced training set, we recommend to selected randomly from the UFPR05 and PUCPR training subsets the same amount of images available on UFPR04, which presents the smallest number of training samples.

The list of proposed research directions is not exhaustive. For instance, another direction using the PKLot could be to investigate throughout an error analysis the real impact on the system performance caused by images from different climatic conditions (sunny, rainy and overcast). With such analysis, it is possible to define new features and parameters to make a system fine tuning. 


\section{Acknowledgments}

This research has been supported by The National Council for Scientific and Technological Development $(\mathrm{CNPq})$ and Araucaria Foundation.

\section{References}

[Almeida et al.(2013)Almeida, Oliveira, Silva, Britto Jr \& Koerich] Almeida, P., Oliveira, L. S., Silva, E., Britto Jr, A. S., \& Koerich, A. (2013). Parking space detection using textural descriptors. In Systems, Man, and Cybernetics (SMC), 2013 IEEE International Conference on (pp. 3603-3608). doi:10.1109/SMC.2013.614. 